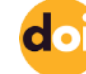

http://doi.org/10.22282/ojrs.2021.91

\title{
EXAMINATION OF THE AFFECT OF THE QUARANTINE DURING THE SARS-COV-2 PANDEMIC ON BODY COMPOSITION VALUES
}

\author{
AHMET KASIK ${ }^{1 *}$, MEHMET CEVAT TEMIZKAN ${ }^{2}$, DOGUKAN OZEN ${ }^{3}$ \\ ${ }^{1}$ Department of Physical Education and Sports, Institute of Graduate Programes, \\ Bozok University, Yozgat, Turkey \\ ${ }^{2}$ Department of Laboratory and Veterinary Health, Sefaatli Vocational College, \\ Bozok University, Yozgat, Turkey \\ ${ }^{3}$ Department of Biostatistics, Faculty of Veterinary Medicine, Ankara University, \\ Ankara, Turkey Turkey
}

*Corresponding Author: Ahmet KASIK. Department of Phycical Education and Sports, Institute of Graduate Programes, Bozok University, Erdogan Akdag Kampusu Ataturk Yolu 7. km 66100 Saglik Bilimleri Fakultesi Dekanligi Binasi Kat: 3, Yozgat, Turkey; Tel: +903545644007; e-mail address: ahmet.kasik@yobu.edu.tr

\section{ABSTRACT}

Problem Statement: Staying and working at home due to SARS-CoV-2 pandemic, caused a lack of physical activity and lead people to be more inactive and incent to consume more unhealty food during the day comparing with pre-pandemic. Balanced nutrition and physical activity are important in terms of keeping body composition values at optimum and therefore staying healthy. Approach: Many factors exist that could affect body composition values, however, balanced nutrition and physical activity are foremost effective. These, in particular, affect the body's fat-muscle ratios and body mass index (BMI). Purpose: In the study, it was aimed to examine the relationship between body composition values and nutrition and physical activities of the three-month quarantine period due to the SARS-CoV-2 (Covid-19) pandemic. For this purpose, body composition values of 16 subjects before and after the quarantine period were evaluated in terms of weight, body fat mass, total body water, mass out of fat, visceral fat, obesity, body mass index, muscle mass. Results: Nutrition and physical activity data of the subjects were determined by examining the questionnaire applied to the subjects. A significant correlation $(p<0.05$ ) was found between the obesity value and the subjects who consumed the same or less food during quarantine comparing with pre-pandemic. Moreover, it was observed that there were statistically significant decreases $(p<0.05)$ in the weight, body fat mass, obesity and visceral fat values, and a remarkable decrease $(p<0.001)$ in $\mathrm{BMI}$ value of the subjects who had to work during the quarantine. Conclusions: Hereby; it is seen that stable nutrition and work-life positively affect body composition values. It was determined that specifically, the working subjects ameliorate their body composition values, thus may have better health.

Key Words: Body composition values, covid-19, nutrition, physical activity, sars-cov-2, quarantine 


\section{INTRODUCTION}

The SARS-CoV-2 (Covid-19) outbreak, which started in Wuhan, China in December 2019, quickly spread to the entire world. It was expected to remain epidemic such as SARS-CoV or MERS-CoV (Drosten et al., 2003; Zaki et al., 2012), however, SARS-COV-2 rapidly turned out to pandemic which leads many countries to take rapid measures. The measures aim to minimize the risk of transmission began with the mask-wearing exigency, then evolve to curfew and quarantines (WHO, 2020). The first case was seen in Turkey on 11 March 2020 as part of this process, a number of measures were taken and restrictions swiftly began. Thereupon the restrictions, warnings and fear of the people, the general public stayed at home for three months and did not leave their homes until the decrease of restrictions due to the downturn in the number of cases (MOH, 2020; MOIA; 2020).

Staying and working at home due to restrictions, caused a lack of physical activity involuntarily. This causes people to be more inactive and incent to consume more unhealty food during the day comparing with prepandemic (Ammar et al., 2020; Blaszczyk-Bebenek et al., 2020; Carter et al., 2020). Balanced nutrition and physical activity are important in terms of keeping body composition values at optimum and therefore staying healthy (Ammar et al., 2020; Chen et al., 2020; Martinez-Ferran et al., 2020; Narici et al., 2020).

The importance of balanced nutrition for health is selfevident (Ammar et al., 2020; Blaszczyk-Bebenek et al., 2020; Zachary et al., 2020). Nutrition, which is one of the fundamental instinct of humans; is taking the essential nutrients into the body at regular intervals and sufficient levels to grow and develop and to continue their life in a healthy way. Adequate and balanced food consumption is required for a healthy diet. If the energy taken into the body is more than the energy that the body burns, the excess energy is stored as fat. Therefore, it negatively affects body composition, especially with fat-related values. Eventually, a number of problems may arise such as weight, obesity and visceral fatness. To prevent this condition, the food variety should be abundant and balanced (Butler and Barrientos, 2020; Carter et al., 2020; Martinez-Ferran et al., 2020; Narici et al., 2020).

On the other hand, physical activity is another event that ensures the continuity of life. A short walk, a short workout and even cleaning can be evaluated within the scope of physical activity. To summarize physical activity; is the whole movements, works and actions in routine life. In this sense, every activity that ends with the use of energy by the organism is considered as physical activity (Aykin and Bilir, 2017). To exemplify, working is a physical activity in itself. Contrary to this, activities such as sitting and sleeping can be considered as inactive/sedentary times without physical activity. It is inevitable that physical activity also affects body composition values, as it is associated with energy consumption (Chastin et al., 2012; Chen et al., 2020; Narici et al., 2020; Zachary et al., 2020).

Many factors exist that could affect body composition values, however, balanced nutrition and physical activity are foremost effective. These, in particular, affect the body's fat-muscle ratios and body mass index (BMI). Also, in the formation of body composition; fats, bones, muscles, extracellular fluids and other organic substances 
such as minerals and proteins are present in the organism (Carter et al., 2020; Hu, 2008; Kyle et al., 2003). Changes in these values are directly related to health (Carter et al., 2020; Chastin et al., 2012; Chen et al., 2020). In the quarantine and restrictions processes that occur due to the SARS-CoV-2 pandemic, the routine life patterns of people frankly changed (Bhutani and Cooper, 2020) and it has been considered that this circumstance may also affect body composition values, especially related to nutrition and physical activity.

This study aims to reveal how staying at home during quarantine affects the relationship between nutrition and physical activities and body composition values. The four essential hypotheses established for this aim are as follows; subjects who consume the same or less food during the quarantine than the pre-pandemic period should have better body composition values than those who are consuming more, subjects who had to work during the quarantine should have better body composition values compared to the subjects who do not work, subjects who engage in any physical activity even for a short time during the quarantine, should have better body composition values, subjects who are more active/less sedentary during the quarantine should have better body composition values compared to the subjects who are less active.

\section{MATERIAL \& METHODS}

\section{Ethics Statement}

The research protocol of the current study was approved by the Turkish Republic Ministry of Health (No. T14_52_01) and Yozgat Bozok University Ethics Committee for the Non-Interventional Clinical Research (No. 2020-12-235). Also, the informed consent has been signed by the clinic and all human subjects who have supported the study.

\section{Timing and Test Subject Selection}

In the study; the declaration of restrictions in Turkey and the date of began to reduce the quarantine measures, the 3-month period between 15th March 2020 and 15th June 2020 was taken into consideration. It was paid regard to the subjects included in the study who did not have any chronic diseases or health problems during this period according to clinical health records. As a result, it was decided to use 16 subjects in the present work. 12 male and 4 female subjects, the age between 19-37 and the lenght between 159-183 cm participants were participated in the experiment.

\section{Nutritional and Physical Activity Data Collection}

Retrospective records for the food consumption data and daily routine information, questionnaire were used to determine the nutritional and physical activity values of the subjects to understand the effect of the differences before and after pandemic restrictions. To obtain the nutrition and physical activities of the subjects, the questions specified in Table 1 were asked to the participants who participated in the experiment. 


\section{Body Composition Analysis}

Within the specified period of three-months, the comparative analyzes of the body compositions before the pandemic and after quarantine restrictions were evaluated. The evaluation was carried out retrospectively with clinical data. Analyzes were performed with Tanita BC 601 device. During the use of the device, all subjects were wearing light clothes, with all their belongings were left and their feet were bare. The analysis was completed with bioelectric resistance measurements in 30 seconds with $50 \mathrm{kHz}$ current and 0.8mA (Ozer, 2009). At the end of the period, the subjects were analyzed in terms of Weight, Body Fat Mass, Total Body Water, Mass out of Fat, Visceral Fat, Muscle Mass, Obesity, Body Mass Index (BMI).

\section{Statistical Analysis}

Descriptive statistics for each variable were calculated and presented as "Mean \pm Standard Error of Mean" and "Median (Range)" for continuous data and frequencies (n) with percentages (\%) for categorical data. Before performing the statistical analysis, data was examined with Shapiro-Wilk test for normality and Levene test for homogeneity of variances as parametric test assumptions. Data were subjected to two-way mixed ANOVA (Analysis of Variance) using General Linear Model for repeated measurements procedure to examine the differences between the "Group" and "Sampling Time" of the measurements obtained. The model included "Group" and "Time" as the main effects and "Group x Time" interaction effects. Simple effect analysis with Bonferroni adjustment was used to break down the significant interaction effect term. In cases where the test assumptions are violated, a robust two-way mixed ANOVA procedure was used. A probability value of less than 0.05 was considered significant unless otherwise noted. Stata 16.1 and R v 4.0.2 (WRS2 package) was used for statistical analysis (StataCorp, 2019; R Core Team, 2020; Mair and Wilcox, 2020).

TABLE 1. NUTRITIONAL AND PHYSICAL ACTIVITY QUESTIONNAIRE

\begin{tabular}{|l|l|}
\hline \multicolumn{1}{|c|}{ Questions } & \multicolumn{1}{|c|}{ Answers } \\
\hline $\begin{array}{l}\text { Q1. What was the amount of food you consume during the pandemic } \\
\text { compared to the pre-pandemic period? }\end{array}$ & $\begin{array}{l}\text { A) Same or less than pre-pandemic } \\
\text { B) More than pre-pandemic }\end{array}$ \\
\hline $\begin{array}{l}\text { Q2. During the pandemic, did you have you to worked outside the } \\
\text { home for paid or unpaid work in routine? }\end{array}$ & $\begin{array}{l}\text { A) No } \\
\text { B) Yes }\end{array}$ \\
\hline Q3. During the pandemic, how many days did you have to bike? & $\begin{array}{l}\text { A) I had to (how many days x minutes } \\
\text { or hours) } \\
\text { B) I didn't have to }\end{array}$ \\
\hline $\begin{array}{l}\text { Q4. During the pandemic, how many days did you have to walk for } \\
\text { more than 10 minutes? }\end{array}$ & $\begin{array}{l}\text { A) I had to (how many days x minutes } \\
\text { or hours) } \\
\text { B) I didn't have to }\end{array}$ \\
\hline $\begin{array}{l}\text { Q5. During the pandemic, how many days did you have to do do } \\
\text { housework (sweeping floors, wiping windows, etc.) for more than 10 } \\
\text { minutes? }\end{array}$ & $\begin{array}{l}\text { A) I had to (how many days x minutes } \\
\text { or hours) } \\
\text { B) I didn't have to }\end{array}$ \\
\hline $\begin{array}{l}\text { Q6. During the pandemic, how many days have you been in sports } \\
\text { activities (swimming, playing football, tennis, etc.) for more than 10 } \\
\text { minutes? }\end{array}$ & $\begin{array}{l}\text { A) I did (how many days x minutes or } \\
\text { hours } \\
\text { B) I didn't }\end{array}$ \\
\hline
\end{tabular}


Q7. How many hours of your day, do you spend sitting and / or sleeping during the pandemic?
A) Less than 18 hours

B) 18 hours or more

\section{RESULTS}

\section{Nutritional and Physical Activity Results}

Nutritional and physical activity analysis results were evaluated in detail in Table 2, according to the results of the questionnaire. It was found that 8 subjects out of 16 consumed more food during the quarantine period compared to the pre-pandemic period, and 8 subjects consumed the same or less food compared to the pre-pandemic period. For physical activity values; it was understood that 8 of 16 subjects had to work during the quarantine period and 8 subjects did not work at all. Equivalently, it was observed that 8 subjects out of 16 remained inactive for less than 18 hours during the quarantine period, and 8 subjects remained inactive for more than 18 hours. On the other hand, it appears that no subject used a bicycle during the quarantine. In other physical activity analysis results, the ratio between the number of subjects is not the same as food consumption, working or activation during the day, it is seen that there are small differences between the groups.

\section{Body Composition Analysis Results}

Detailed analysis data on body composition values before and after quarantine were presented in Table 3. When the changes in the pre-pandemic and post-quarantine periods were examined without any grouping, it was seen that there was no statistically significant difference between body composition values (data not shown).

\section{Comperative Results}

Along with the question number one asked in the questionnaire (Q1), it was aimed to investigate the effects of the nutritional status of the subjects on body composition. It was found in this study; there were statistically significant decreases in obesity values after quarantine for the group who consume the same or less food during quarantine (Table 4). There were also notable differences in obesity values for the subjects who answered this question as "I consumed more food during quarantine" and "I consumed the same or less food during quarantine". Nonetheless, in this study; no significant effect of nutrition on body composition values except obesity was found.

Further, with question number two asked in the questionnaire (Q2), it was intended to examine the effects of the working status of the subjects on body composition. It has been observed that the subjects who had to work during quarantine, the weight, body fat mass, obesity, BMI and visceral fat values decrease significantly (Table 5). Especially, BMI value decrease was remarkable $(p<0,001)$. No noticeable difference was found in any of these values for subjects who did not work during the quarantine period. Therewithal, for weight and visceral fat values, there are significant differences between subjects who have to work during quarantine and those who do not. Except for these results, no noteworthy difference was found for the total body water, mass out of fat and muscle mass values for Q2. 
In the comparison of Q3, Q4, Q5, Q6 and Q7 physical activity data with body composition values, no notable associations were found between body composition values and physical activities such as walking, houseworking or sedentary such as sitting or sleeping (data not shown).

Table 2. Nutritıonal and physıcal actıvity descriptıve statıstıcs results

\begin{tabular}{|c|c|c|c|c|c|c|}
\hline Questions & Category & $\mathbf{n}$ & Details & $\begin{array}{l}\text { Arithmetic } \\
\text { Mean } \pm \\
\text { Standard } \\
\text { Error }\end{array}$ & $\begin{array}{l}\text { Standard } \\
\text { Deviation }\end{array}$ & $\begin{array}{c}\text { Median } \\
\text { (Min - } \\
\text { Max) }\end{array}$ \\
\hline \multirow[t]{2}{*}{ Q1 } & $\begin{array}{l}\text { Same or less than pre- } \\
\text { pandemic }\end{array}$ & $8(\% 50)$ & & & & \\
\hline & More than pre-pandemic & $8(\% 50)$ & & & & \\
\hline \multirow{2}{*}{ Q2 } & No & $8(\% 50)$ & & & & \\
\hline & Yes & $8(\% 50)$ & & & & \\
\hline \multirow{2}{*}{ Q3 } & I didn't have to & $16(\% 100)$ & & & & \\
\hline & I had to & $0(\% 0)$ & & & & \\
\hline \multirow{2}{*}{ Q4 } & I didn't have to & $7(\% 43.8)$ & & & & \\
\hline & I had to & $9(\% 56.3)$ & $\begin{array}{c}\text { days } \mathrm{x} \text { minutes or } \\
\text { hours }\end{array}$ & $27.28 \pm 8.33$ & 24.98 & $\begin{array}{c}30 \\
(0.5-75)\end{array}$ \\
\hline \multirow{2}{*}{ Q5 } & I didn't have to & $7(\% 43.8)$ & & & & \\
\hline & I had to & $9(\% 56.3)$ & $\begin{array}{c}\text { days } \mathrm{x} \text { minutes or } \\
\text { hours }\end{array}$ & $20.64 \pm 7.95$ & 23.84 & $\begin{array}{c}8 \\
(3.75-75) \\
\end{array}$ \\
\hline \multirow{2}{*}{ Q6 } & I didn't & $10(\% 62.5)$ & & & & \\
\hline & I did & $6(\% 37.5)$ & $\begin{array}{c}\text { days } \mathrm{x} \text { minutes or } \\
\text { hours }\end{array}$ & $1.41 \pm 0.42$ & 1.03 & $\begin{array}{c}1.08 \\
(0.66-3.3)\end{array}$ \\
\hline \multirow{2}{*}{ Q7 } & Less than 18 hours & $8(\% 50)$ & & & & \\
\hline & 18 hours or more & $8(\% 50)$ & & & & \\
\hline
\end{tabular}

Table 3. Pre-pandemıc and after-quarantıne body composıtıon analysıs descrıptıve statıstıcs results

\begin{tabular}{|c|l|c|c|c|c|}
\hline \multirow{4}{*}{ Time } & \multicolumn{1}{|c|}{ Category } & $\mathbf{n}$ & $\begin{array}{c}\text { Arithmetic Mean } \pm \\
\text { Standard Error }\end{array}$ & $\begin{array}{c}\text { Standard } \\
\text { Deviation }\end{array}$ & $\begin{array}{c}\text { Median } \\
\text { (Min - Max) }\end{array}$ \\
\hline \multirow{5}{*}{ Pre-Pandemic } & Weight (kg) & 16 & $70.15 \pm 4.01$ & 16,02 & $70.75(46.3-107.9)$ \\
\cline { 2 - 6 } & Body Fat Mass (kg) & 16 & $13.92 \pm 1.87$ & 7,5 & $12.91(5.84-31.08)$ \\
\cline { 2 - 6 } & Total Body Water (kg) & 16 & $40.44 \pm 1.92$ & 7,67 & $40.75(27.47-53.09)$ \\
\cline { 2 - 6 } & Mass out of Fat (kg) & 16 & $56.18 \pm 2.86$ & 11,43 & $56.65(37.4-76.8)$ \\
\cline { 2 - 6 } & Muscle Mass (kg) & 16 & $53.42 \pm 2.72$ & 10,89 & $53.9(35.5-73.1)$ \\
\cline { 2 - 6 } & Obesity (\%) & 16 & $6.24 \pm 4.54$ & 18,17 & $3.13(-15.51-55.27)$ \\
\cline { 2 - 6 } & Body Mass Index & 16 & $23.57 \pm 0.92$ & 3,67 & $23.61(18.35-32.22)$ \\
\cline { 2 - 6 } & Mineral (\%) & 16 & $3.83 \pm 0.25$ & 1 & $4.04(1.95-5.61)$ \\
\cline { 2 - 6 } & Protein (\%) & 16 & $11.96 \pm 0.74$ & 2,96 & $12.12(7.87-18.13)$ \\
\cline { 2 - 6 } After & Visceral Fat & 16 & $4.13 \pm 0.72$ & 2,87 & $4(1-10)$ \\
\hline \multirow{5}{*}{\begin{tabular}{l} 
Quarantine \\
\cline { 2 - 6 }
\end{tabular}} & Weight (kg) & 16 & $69.77 \pm 4.04$ & 16,15 & $69.1(45.4-108.2)$ \\
\cline { 2 - 6 } & Body Fat Mass (kg) & 16 & $13.31 \pm 1.87$ & 7,5 & $11.18(5.72-31.75)$ \\
\cline { 2 - 6 } & Total Body Water (kg) & 16 & $40.45 \pm 2$ & 7,99 & $41.31(26.28-54.42)$ \\
\cline { 2 - 6 } & Mass out of Fat (kg) & 16 & $56.42 \pm 3.01$ & 12,04 & $57.5(35.8-78.5)$ \\
\cline { 2 - 6 } & Muscle Mass (kg) & 16 & $53.64 \pm 2.86$ & 11,46 & $54.65(34-74.7)$ \\
\cline { 2 - 6 } & Obesity (\%) & 16 & $5.12 \pm 4.66$ & 18,63 & $0.77(-17.09-54)$ \\
\cline { 2 - 6 } & Body Mass Index & 16 & $23.42 \pm 0.91$ & 3,64 & $23.04(18.66-31.96)$ \\
\cline { 2 - 6 } & Mineral (\%) & 16 & $4.07 \pm 0.24$ & 0,98 & $4.2(1.86-5.73)$ \\
\cline { 2 - 6 } & Protein (\%) & 16 & $11.94 \pm 0.78$ & 3,12 & $12.06(7.7-18.4)$ \\
\cline { 2 - 6 } & Visceral Fat & 16 & $3.75 \pm 0.62$ & 2,46 & $4(1-9)$ \\
\hline
\end{tabular}


TABLE 4. Pre-pandemıc and after-quarantıne comperatıve statıstıcal analysıs for food consumptıon

\begin{tabular}{|c|c|c|c|c|c|c|c|}
\hline \multirow[t]{3}{*}{ Group Q1 } & $\begin{array}{l}\text { Arithme } \\
\text { tic } \\
\text { Mean } \pm \\
\text { Standar } \\
\text { d Error }\end{array}$ & $\begin{array}{l}\text { Median } \\
\text { (Range) }\end{array}$ & $\begin{array}{l}\text { Arithmetic } \\
\text { Mean } \pm \\
\text { Standard } \\
\text { Error }\end{array}$ & $\begin{array}{l}\text { Median } \\
\text { (Range) }\end{array}$ & \multirow{2}{*}{\multicolumn{3}{|c|}{$\mathbf{p}$}} \\
\hline & \multicolumn{2}{|c|}{ Pre-Pandemic } & \multicolumn{2}{|c|}{ After-Quarantine } & & & \\
\hline & \multicolumn{4}{|c|}{ Weight } & Time & Group & $\begin{array}{l}\text { Time } x \\
\text { Group }\end{array}$ \\
\hline $\begin{array}{l}\text { Same or less than pre- } \\
\text { pandemic }\end{array}$ & $\begin{array}{c}65,02 \pm \\
3,96 \\
\end{array}$ & $66,5(34,6)$ & $\begin{array}{c}63,94 \pm \\
3,73 \\
\end{array}$ & $\begin{array}{l}65,25 \\
(34,4) \\
\end{array}$ & \multirow[t]{2}{*}{0,397} & \multirow[t]{2}{*}{0,181} & \multirow[t]{2}{*}{0,119} \\
\hline More than pre-pandemic & $\begin{array}{c}75,28 \pm \\
6,75\end{array}$ & $76,4(61,5)$ & $75,61 \pm 6,8$ & $\begin{array}{l}76,25 \\
(61,1) \\
\end{array}$ & & & \\
\hline & \multicolumn{4}{|c|}{ Body Fat Mass } & & & \\
\hline $\begin{array}{l}\text { Same or less than pre- } \\
\text { pandemic }\end{array}$ & $\begin{array}{c}10,06 \pm \\
1,27\end{array}$ & $9,46(9,35)$ & $8,87 \pm 0,81$ & $8,94(5,86)$ & \multirow[t]{2}{*}{0.24} & \multirow[t]{2}{*}{0,019} & \multirow[t]{2}{*}{0,264} \\
\hline More than pre-pandemic & $\begin{array}{c}17,78 \pm \\
3,03\end{array}$ & $\begin{array}{c}13,76 \\
(22,12)\end{array}$ & $\begin{array}{c}17,75 \pm \\
2,96\end{array}$ & $\begin{array}{c}14,48 \\
(21,15)\end{array}$ & & & \\
\hline & \multicolumn{4}{|c|}{ Total Body Water } & & & \\
\hline $\begin{array}{l}\text { Same or less than pre- } \\
\text { pandemic }\end{array}$ & $\begin{array}{c}39,41 \pm \\
2,15\end{array}$ & $\begin{array}{c}40,34 \\
(20,35)\end{array}$ & $\begin{array}{c}39,51 \pm \\
2,13\end{array}$ & $\begin{array}{c}40,39 \\
(20,37)\end{array}$ & \multirow[t]{2}{*}{0,984} & \multirow[t]{2}{*}{0,631} & \multirow[t]{2}{*}{0,742} \\
\hline More than pre-pandemic & $\begin{array}{c}41,48 \pm \\
3,29\end{array}$ & $\begin{array}{c}44,11 \\
(25,62)\end{array}$ & $\begin{array}{c}41,39 \pm \\
3,51\end{array}$ & $\begin{array}{c}43,58 \\
(28,14)\end{array}$ & & & \\
\hline & \multicolumn{4}{|c|}{ Mass out of Fat } & & & \\
\hline $\begin{array}{l}\text { Same or less than pre- } \\
\text { pandemic }\end{array}$ & $\begin{array}{c}54,9 \pm \\
3,18\end{array}$ & $56,3(30)$ & $\begin{array}{c}55,03 \pm \\
3,14\end{array}$ & $56,5(30)$ & \multirow[t]{2}{*}{0,459} & \multirow[t]{2}{*}{0,664} & \multirow[t]{2}{*}{0,724} \\
\hline More than pre-pandemic & $\begin{array}{c}57,46 \pm \\
4,94\end{array}$ & $\begin{array}{l}61,15 \\
(39,4)\end{array}$ & $\begin{array}{c}57,81 \pm \\
5,33\end{array}$ & $61,2(42,7)$ & & & \\
\hline & \multicolumn{4}{|c|}{ Muscle Mass } & & & \\
\hline $\begin{array}{l}\text { Same or less than pre- } \\
\text { pandemic }\end{array}$ & $\begin{array}{c}52,21 \pm \\
3,04\end{array}$ & $\begin{array}{l}53,55 \\
(28,7)\end{array}$ & $52,3 \pm 2,99$ & $53,7(28,5)$ & \multirow[t]{2}{*}{0,477} & \multirow[t]{2}{*}{0,664} & \multirow[t]{2}{*}{0,668} \\
\hline \multirow[t]{2}{*}{ More than pre-pandemic } & $\begin{array}{c}54,63 \pm \\
4,7\end{array}$ & $58,1(37,6)$ & $\begin{array}{c}54,97 \pm \\
5,07\end{array}$ & $58,2(40,7)$ & & & \\
\hline & \multicolumn{4}{|c|}{ Obesity } & & & \\
\hline $\begin{array}{l}\text { Same or less than pre- } \\
\text { pandemic }\end{array}$ & $\begin{array}{c}- \\
3,37 \pm 3,0 \\
8 a, B\end{array}$ & $\begin{array}{c}-2,94 \\
(23,68)\end{array}$ & $\begin{array}{c}-6,05 \pm 2,66 \\
b, B\end{array}$ & $\begin{array}{c}-5,05 \\
(20,73)\end{array}$ & \multirow[t]{2}{*}{0,067} & \multirow[t]{2}{*}{0,017} & \multirow[t]{2}{*}{$0,015^{*}$} \\
\hline More than pre-pandemic & $\begin{array}{c}15,85 \pm 7 \\
2 a, A\end{array}$ & $\begin{array}{l}18,66 \\
(69,9) \\
\end{array}$ & $\begin{array}{c}16,3 \pm 7,08 \\
a, A\end{array}$ & $\begin{array}{c}19,24 \\
(66,24) \\
\end{array}$ & & & \\
\hline & \multicolumn{4}{|c|}{ Body Mass Index (BMI) } & & & \\
\hline $\begin{array}{l}\text { Same or less than pre- } \\
\text { pandemic }\end{array}$ & $\begin{array}{c}22,01 \pm \\
0,78\end{array}$ & $21,91(6,4)$ & $\begin{array}{c}21,61 \pm \\
0,63\end{array}$ & $21,68(5,3)$ & \multirow[t]{2}{*}{0,291} & \multirow[t]{2}{*}{0,062} & 0,09 \\
\hline More than pre-pandemic & $\begin{array}{c}25,13 \pm \\
1,52 \\
\end{array}$ & $\begin{array}{c}24,62 \\
(13,87) \\
\end{array}$ & $\begin{array}{c}25,23 \pm \\
1,49 \\
\end{array}$ & $\begin{array}{c}24,79 \\
(13,09) \\
\end{array}$ & & & \\
\hline & & Visc & ral Fat & & & & \\
\hline $\begin{array}{l}\text { Same or less than pre- } \\
\text { pandemic }\end{array}$ & $\begin{array}{c}3,75 \pm \\
0,8 \\
\end{array}$ & $4(6)$ & $3,13 \pm 0,58$ & $3,5(4)$ & 0,138 & 0,467 & 0,312 \\
\hline More than pre-pandemic & $\begin{array}{l}4,5 \pm \\
1,24\end{array}$ & $3,5(9)$ & $4,38 \pm 1,08$ & $4(8)$ & & & \\
\hline
\end{tabular}

*a,b: Different letters in a same row are significantly different from one another $(p<0.05)$

*A,B: Different letters in a same column are significantly different from one another $(p<0.05)$ 
Table 5. Pre-pandemıc and after-quarantıne comperatıve statıstıcal analysıs for workıng and non-workıng subjects

\begin{tabular}{|c|c|c|c|c|c|c|c|}
\hline \multirow{3}{*}{$\begin{array}{c}\text { Group } \\
\text { Q2 }\end{array}$} & $\begin{array}{l}\text { Arithmetic Mean } \\
\pm \text { Standard Error }\end{array}$ & $\begin{array}{l}\text { Median } \\
\text { (Range) }\end{array}$ & $\begin{array}{l}\text { Arithmetic Mean } \\
\pm \text { Standard Error }\end{array}$ & Median (Range) & \multirow{2}{*}{\multicolumn{3}{|c|}{$\mathbf{p}$}} \\
\hline & \multicolumn{2}{|c|}{ Pre-Pandemic } & \multicolumn{2}{|c|}{ After-Quarantine } & & & \\
\hline & \multicolumn{4}{|c|}{ Weight } & Time & Group & $\begin{array}{l}\text { Time } x \\
\text { Group }\end{array}$ \\
\hline No & $64,75 \pm 5,53 a, B$ & $63,5(42,7)$ & $65,59 \pm 5,74 a, B$ & $65,05(44,1)$ & \multirow{2}{*}{0,289} & \multirow{2}{*}{0,245} & \multirow{2}{*}{$0,003 *$} \\
\hline \multirow[t]{2}{*}{ Yes } & $75,55 \pm 5,47 a, A$ & $73,7(54,5)$ & $73,96 \pm 5,65 \boldsymbol{b}, \boldsymbol{A}$ & $71,2(55,2)$ & & & \\
\hline & \multicolumn{4}{|c|}{ Body Fat Mass } & & & \\
\hline No & $13,02 \pm 2,95 a, A$ & $10,06(24,32)$ & $13,63 \pm 2,96 a, A$ & $10,93(25,12)$ & \multirow{2}{*}{0,156} & \multirow{2}{*}{0,88} & \multirow{2}{*}{$0,01 *$} \\
\hline \multirow[t]{2}{*}{ Yes } & $14,83 \pm 2,47 a, A$ & $13,38(23,55)$ & $13 \pm 2,51 \boldsymbol{b}, \boldsymbol{A}$ & $11,34(23,93)$ & & & \\
\hline & \multicolumn{4}{|c|}{ Total Body Water } & & & \\
\hline No & $37,64 \pm 2,95$ & $37,16(21,3)$ & $37,54 \pm 3,16$ & $37,28(24,82)$ & \multirow{2}{*}{0,984} & \multirow{2}{*}{0,149} & \multirow{2}{*}{0,732} \\
\hline \multirow[t]{2}{*}{ Yes } & $43,25 \pm 2,19$ & $42,51(19,66)$ & $43,36 \pm 2,18$ & $42,25(19,86)$ & & & \\
\hline & \multicolumn{4}{|c|}{ Mass out of Fat } & \multirow{3}{*}{0,461} & \multirow{3}{*}{0,128} & \\
\hline No & $51,69 \pm 4,23$ & $50,7(31,2)$ & $51,91 \pm 4,64$ & $51(35,6)$ & & & \multirow{2}{*}{0,969} \\
\hline \multirow[t]{2}{*}{ Yes } & $60,67 \pm 3,36$ & $59,6(31)$ & $60,93 \pm 3,38$ & $59,1(31,3)$ & & & \\
\hline & \multicolumn{4}{|c|}{ Muscle Mass } & & & \\
\hline No & $49,13 \pm 4,02$ & $48,2(29,7)$ & $49,35 \pm 4,41$ & $48,5(33,9)$ & \multirow{2}{*}{0,48} & \multirow{2}{*}{0,128} & \multirow{2}{*}{0,984} \\
\hline \multirow[t]{2}{*}{ Yes } & $57,71 \pm 3,21$ & $56,6(29,6)$ & $57,93 \pm 3,23$ & $56,2(29,9)$ & & & \\
\hline & \multicolumn{4}{|c|}{ Obesity } & \multirow{3}{*}{0,081} & \multirow{3}{*}{0,64} & \\
\hline No & $3,29 \pm 5,5$ & $-0,5(38,53) a, A$ & $3,57 \pm 5,92$ & $-2,1(41,97) a, A$ & & & $0035 *$ \\
\hline Yes & $9,19 \pm 7,47$ & $4,9(70,78) a, A$ & $6,68 \pm 7,56$ & $2,8(70,14) \boldsymbol{b}, \boldsymbol{A}$ & & & $0,035^{*}$ \\
\hline & & Body Ma & ndex (BMI) & & & & \\
\hline No & $22,69 \pm 1,34 a, A$ & $21,48(10,06)$ & $22,95 \pm 1,38 a, A$ & $21,52(9,91)$ & & סרי & \\
\hline Yes & $24,45 \pm 1,26 a, A$ & $23,74(12,37)$ & $23,88 \pm 1,26 \boldsymbol{b}, \boldsymbol{A}$ & $23,34(12,26)$ & $0,1 / 2$ & $0,4 / 9$ & $0,001 *$ \\
\hline & & Vis & al Fat & & & & \\
\hline No & $2,88 \pm 1,06$ & $1,5(8) a, B$ & $3 \pm 0,96$ & $1,5(7) a, B$ & (201 & 012 & \\
\hline Yes & $5,38 \pm 0,8$ & $4,5(7) a, A$ & $4,5 \pm 0,73$ & $4(7) b, A$ & 0,094 & 0,133 & $31^{*}$ \\
\hline
\end{tabular}

*a,b: Different letters in a same row are significantly different from one another $(p<0.05)$

*A,B: Different letters in a same column are significantly different from one another $(p<0.05)$

\section{DISCUSSION}

Studies conducted during the pandemic; it has been concluded that nutrition and physical activity decreased, unhealthy nutrition and sedentary time increased, and this negatively affected human health (Ammar et al., 2020; Bertrand et al., 2020; Blaszczyk-Bebenek et al., 2020; Butler and Barrientos, 2020; Martinez-Ferran et al., 2020; Zachary et al., 2020). Previous studies have stated that obese individuals overcome SARS-CoV-2 disease more severely and the negative effects of obesity values in patients (Cinti et al., 2020; Gualtieri et al., 2020). The data obtained in this study reveal the results expressing a similar circumstance with previous datas. In this study, it was observed that during the quarantine, obesity values decreased significantly in subjects consuming the same or less food than the pre-pandemic period (Table 4). Although a similar trend was observed for other body composition values, the resulting differences were statistically insignificant. Current results support the hypothesis about the subjects who consume the same or less food during the quarantine than the pre-pandemic period should have better body composition values than those who are consume more.

It has been present in previous studies that staying at home inactively increase the risk of fatness and obesity, thus makes the SARS-CoV-2 more serious (Cinti et al., 2020; Favre et al., 2020; Narici et al., 2020). Likewise 
before pandemic, the relationship between physical activity, specifically working and body composition values had similar results in previous studies (Arslan and Ceviz, 2007; Chen et al., 2020; Ekingen, 2014; George and Chandan, 2016; Koner ve Bandyopadhyay, 2017). In a study, body composition values between working and nonworking individuals were examined, and it was found that weight, BMI, body fat mass, muscle mass and total body water values decreased in working individuals (Arslan and Ceviz, 2007). In a study conducted during the pandemic period, it was found that there was a statistically significant difference in BMI values during the quarantine period (Giustino et al., 2020). In another study examining the BMI value for working and non-working individuals, it was found that the BMI value was lower in working individuals (George and Chandan, 2016). Our results were compatible with previous studies for the weight, BMI and body fat mass values, but discordant for the muscle mass and total body water (Table 5). The most important finding of our study was undoubtedly the effect of working in any job on BMI values. Our results indicate the BMI values of subjects who had to work during the quarantine period have remarkably decreased $(p<0,001)$. In the meantime, it is seen that the values of weight, body fat mass, obesity and visceral fat decrease in working subjects. In different studies comparing the weight and total body water values between working and non-working individuals, some study indicate that working individuals have lower weight (Ekingen, 2014), and some studies do not refer to any significance (Damania and Machado, 2014; George and Chandan, 2016). Our results were congruous with the study that indicate significant differences in weight value (Table 5). Further, in another study examining mass out of fat, it was found that there was no difference between working and non-working individuals (Damania and Machado, 2014; Koner ve Bandyopadhyay, 2017). The results of our work have similar results with the previous study (Table 5). Our results also reveal that the working creates positive effects in terms of body composition values. As foreseen in a previous literature (Bhutani and Cooper, 2020), the subjects who partially continue their routine life and work during pandemic, stay healthier than non-working subjects. This confirms our hypothesis about the subjects who had to work during the quarantine should have better body composition values compared to the subjects who do not work. Besides, our results may infer that working has more influence on body composition values than nutrition.

In this research, none of the subjects used bicycles during the quarantine, so no evaluation can be made on this issue. Moreover, no significant results were found between body composition values and physical activities such as short walks, cleaning activities or short sports activities. The reason of it might be the minimal and unequal number of subjects for these analyzes, thus further studies are required. Nevertheless, the number of subjects were equal for active and inactive subjects, it was observed that activation during the day did not have a significant effect on body composition values. Although this result does not definitely reveal the effect of sedentary on body composition values, it suggests that sedentary might less important than working and nutrition. However, more research is required on this issue as well. 


\section{CONCLUSIONS}

The results of this study aid to demonstrate how nutrition and physical activity during quarantine affect body composition values. The results indicate that while nutrition affected obesity, working had a significant effect to reduce weight, body fat mass, obesity, BMI and visceral fat values. Especially the decrease in BMI value was remarkable. It has been understood that working in any job may affect body composition values more than nutrition and some physical activities. Collectively; in the quarantine period, it has been observed that subjects who work and consume the same or less food comparing with pre-pandemic, parry this continuum more healthily. Considering that nutrition and working in the routine life before the pandemic affects most of life, it is seen how important routine life is in terms of body composition values and therefore health. Subjects who continued or had to continue their routine life partially during the quarantine remained healthier.

CONFLICTS OF INTEREST: The authors declare that they have no conflict of interests.

\section{REFERENCES:}

Ammar, A., Brach, M., Trabelsi, K., Chtourou, H., Boukhris, O., Masmoudi, L., Bouaziz, B., Bentlage, E., How, D., Ahmed, M. \& Müller, P. (2020). Effects of COVID-19 Home Confinement on Eating Behaviour and Physical Activity: Results of the ECLB-COVID19 International Online Survey. Nutrients, 12(6), 1583.

Arslan, C., \& Ceviz, D. (2007). Ev hanimi ve calisan kadinlarin obezite prevalansi ve saglikli yasam bicimi davranislarinin degerlendirilmesi. Fırat University Sag. Bil. Derg., 211-220.

Aykin, A. G. \& Bilir, F. P. (2017). Evaluation of the relation between physical activity and quality of life in terms of the sustainable development Sample of Antalya and Osmaniye public education centers. Niğde Üniversitesi Beden Eğitimi ve Spor Bilimleri Dergisi, 11(3), 310-319

Bertrand, L., Shaw, K.A., Ko, J., Deprez, D., Chilibeck, P.D., \& Zello, G.A. (2020). The impact of the coronavirus disease 2019 (COVID-19) pandemic on university students' dietary intake, physical activity, and sedentary behaviour. Applied Physiology, Nutrition, and Metabolism. (Accepted Article).

Bhutani, S., Cooper, J. A. (2020). COVID-19-Related Home Confinement in Adults: Weight Gain Risks and Opportunities. Obesity, 28(9), 1576-1577.

Blaszczyk-Bebenek, E., Jagielski, P., Boleslawska, I., Jagielska, A., Nitsch-Osuch, A., \& Kawalec, P. (2020). Nutrition behaviors in Polish adults before and during covid-19 lockdown. Nutrients, 12(10), 3084.

Butler, M. J., \& Barrientos, R. M. (2020). The impact of nutrition on COVID-19 susceptibility and long-term consequences. Brain, behavior, and immunity. 87, 53-54.

Carter, S. J., Baranauskas, M. N., \& Fly, A. D. (2020). Considerations for obesity, vitamin D, and physical activity amid the COVID-19 pandemic. Obesity, 28(7), 1176-1177.

Chastin, S. F. M., Ferriolli, E., Stephens, N. A., Fearon, K. C., \& Greig, C. (2012). Relationship between sedentary behaviour, physical activity, muscle quality and body composition in healthy older adults. Age and ageing, 41(1), 111-114.

Chen, P., Mao, L., Nassis, G. P., Harmer, P., Ainsworth, B., \& Li, F. (2020). Coronavirus disease (COVID-19): The need to maintain regular physical activity while taking precautions. Journal Of Sport And Health Science, 9, 103-104.

Cinti, S., Graciotti, L., Giordano, A., Valerio, A., \& Nisoli, E. (2020). COVID-19 and fat embolism: a hypothesis to explain the severe clinical outcome in people with obesity. International Journal of Obesity, 44(8), 18001802. 
Damania, H., \& Machado, P. (2014). Nutritional Assessment of Working and Non-Working Mothers: A Pilot Study. Int J Innov Res Dev.

Drosten, C., Günther, S., Preiser, W., Van Der Werf, S., Brodt, H. R., Becker, S., ... \& Berger, A. (2003). Identification of a novel coronavirus in patients with severe acute respiratory syndrome. New England journal of medicine, 348(20), 1967-1976.

Ekingen, A. (2015). Farkli meslek gruplarinda calisan ve calismayan kadinlarin vucut yag yuzdesi uzerine etkisi (Unpublished doctoral dissertation, University of Dicle).

Favre, G., Legueult, K., Pradier, C., Raffaelli, C., Ichai, C., Iannelli, A., Redheuil, A., Lucidarme, O., \& Esnault, V. (2020). Visceral fat is associated to the severity of COVID-19. Metabolism Clinical and Experimental, 115, 154440.

George, S. O., \& Chandan, L. M. (2016). Is the physical fitness of working women better than homemakers? Indian Journal of Basic and Applied Medical Research, 629-636.

Giustino, V., Parroco, A. M., Gennaro, A., Musumeci, G., Palma, A., \& Battaglia, G. (2020). Physical activity levels and related energy expenditure during COVID-19 quarantine among the Sicilian active population: a cross-sectional online survey study. Sustainability, 12(11), 4356.

Gualtieri, P., Falcone, C., Romano, L., Macheda, S., Correale, P., Arciello, P., ... \& Lorenzo, A. D. (2020). Body composition findings by computed tomography in SARS-CoV-2 patients: increased risk of muscle wasting in obesity. International Journal of Molecular Sciences, 21(13), 4670.

Hu, F. B. (2008). Measurements of adiposity and body composition. Obesity epidemiology, 416, 53-83.

Koner, D., \& Bandyopadhyay, P. S. (2017). Health fitness of mid age working women and non-working house wiwes - a comparative study. Indian Journal Of Applied Research, 383-384.

Kyle, U. G., Schutz, Y., Dupertuis, Y. M., \& Pichard, C. (2003). Body composition interpretation: contributions of the fat-free mass index and the body fat mass index. Nutrition, 19(7-8), 597-604.

Mair., P., \& Wilcox., R. (2020). Robust Statistical Methods in R Using the WRS2 Package. Behavior Research Methods, 52, 464-488.

Martinez-Ferran, M., de la Guía-Galipienso, F., Sanchis-Gomar, F., \& Pareja-Galeano, H. (2020). Metabolic impacts of confinement during the COVID-19 pandemic due to modified diet and physical activity habits. Nutrients, 12(6), 1549.

MOH (2020). Turkish Republic Ministry of Health. Source: [https://www.saglik.gov.tr/]

MOIA (2020). Turkish Republic Ministry of Internal Affairs. Source: [https://www.icisleri.gov.tr/]

Narici, M., De Vito, G., Franchi, M., Paoli, A., Moro, T., Marcolin, G., Grassi B., Baldassarre G., Zuccarelli L., Biolo G., Girolamo F.G., Fiotti N., Dela F., Greenhaff, P., \& Maganaris, C. (2020). Impact of sedentarism due to the COVID-19 home confinement on neuromuscular, cardiovascular and metabolic health: Physiological and pathophysiological implications and recommendations for physical and nutritional countermeasures. European journal of sport science, 1-22.

Ozer, P.D. (2009). Kinantropometri, Sporda Morfolojik Planlama. Ankara: Nobel Yayin Dagitim.

R Core Team (2020). R:A Language and Environment for Statistical Computing. R Foundation for Statistical Computing, Vienna, Austria.

StataCorp. 2019. Stata Statistical Software: Release 16. College Station, TX: StataCorp LLC.

WHO (2020). World Health Organization. Source: [https://www.who.int/].

Zachary, Z., Brianna, F., Brianna, L., Garrett, P., Jade, W., Alyssa, D., \& Mikayla, K. (2020). Self-quarantine and weight gain related risk factors during the COVID-19 pandemic. Obesity research \& clinical practice, $14(3), 210-216$.

Zaki, A. M., Van Boheemen, S., Bestebroer, T. M., Osterhaus, A. D., \& Fouchier, R. A. (2012). Isolation of a novel coronavirus from a man with pneumonia in Saudi Arabia. New England Journal of Medicine, 367(19), 1814-1820. 